\title{
IMPACTO DE MICRONUTRIENTES EN POLVO SOBRE LA ANEMIA EN NIÑOS DE 10 A 35 MESES DE EDAD EN APURIMMAC, PERÚ
}

\author{
Juan Pablo Aparco ${ }^{1,2, a}$, Luz Bullón"2,3,b, Sandra Cusirramos ${ }^{4, c}$
}

\begin{abstract}
RESUMEN
Objetivos. Evaluar el impacto de la suplementación con micronutrientes en polvo (MNP) en la reducción de anemia en niños de 10 a 35 meses de edad de Apurímac, Perú. Materiales y métodos. Se realizó un análisis secundario de la base de datos de la Evaluación de suplementación con MNP en la región de Apurímac, realizada por el Centro Nacional de Alimentación y Nutrición (CENAN) entre 2009 y 2010. Se definió como grupo de intervención a los niños que consumieron 60 o más sobres de MNP, para generar los controles (niños sin consumo de MNP) aplicamos un propensity score matching (PSM), y en la estimación del impacto de los MNP comparamos la prevalencia de anemia en cada grupo de estudio, ponderada por la probabilidad de participación, aplicando tres algoritmos de emparejamiento: vecino más cercano, kernel y regresión lineal local. Todos los cálculos se realizaron en el programa estadístico Stata 14 SE. Resultados. La prevalencia de anemia fue significativamente menor hasta en 11 puntos porcentuales en el grupo de intervención comparado con los controles $(p=0,001)$ y que el promedio de hemoglobina aumentó en $0,3 \mathrm{~g} / \mathrm{dL}$ en el mismo grupo $(p<0,001)$. Conclusiones. La suplementación con MNP mostró impacto para reducir la anemia e incrementar el nivel de hemoglobina en niños que consumieron 60 o más sobres de MNP en un periodo de seis meses. Se deben continuar los esfuerzos por reducir la anemia empleando los MNP para prevenir esta deficiencia nutricional.
\end{abstract}

Palabras clave: Anemia; Hierro; Micronutrientes; Evaluación del Impacto en la Salud; Niño (fuente: DeCS BIREME).

\section{IMPACT OF MICRONUTRIENT POWDER ON ANEMIA IN CHILDREN AGED 10-35 MONTHS IN APURIMAC, PERU}

\begin{abstract}
Objective. Objective. To assess the impact of micronutrient powder supplementation (MNP) in reducing anemia in children aged 10 to 35 months in Apurimac, Peru. Materials and Methods. A secondary analysis of the database of the Evaluation of supplementation with MNP in the Apurímac region, conducted by the National Food and Nutrition Center (CENAN, in Spanish) between 2009 and 2010, was conducted. The intervention group was defined as children who consumed 60 or more packets of MNP; to generate the controls (children who did not consume MNP), we applied a propensity score matching (PSM); and, to estimate the impact of the MNP, we compared the prevalence of anemia in each study group, weighted by the probability of participation, applying three matching algorithms: nearest neighbor, kernel and local linear regression. All calculations were performed in the Stata 14 SE statistical program. Results. The prevalence of anemia was significantly lower by up to 11 percentage points in the intervention group compared to controls $(p=0.001)$, and mean hemoglobin increased by $0.3 \mathrm{~g} / \mathrm{dL}$ in the same group $(p<0.001)$. Conclusions. MNP supplementation showed impact in reducing anemia and increasing hemoglobin level in children who consumed 60 or more packets of MNP over a six-month period. Efforts to reduce anemia by using MNP to prevent this nutritional deficiency should continue.
\end{abstract}

Keywords: Anemia; Iron; Micronutrients; Health impact assessment; Child (source: MeSH NLM).

\footnotetext{
Centro Nacional de Alimentación y Nutrición, Instituto Nacional de Salud. Lima, Perú.

Escuela de Posgrado, Universidad Nacional Agraria La Molina. Lima, Perú.

Departamento de Estadística e Informática, Universidad Nacional Agraria La Molina. Lima, Perú.

Carrera de Nutrición y Dietética, Universidad Peruana de Ciencias Aplicadas. Lima, Perú.

a Licenciado en Nutrición, magíster en Nutrición Pública; ${ }^{b}$ ingeniero estadístico, magíster en Estadística; clicenciada en Nutrición Humana, magíster en Nutrición Pública.

El presente estudio forma parte de la tesis de Juan Pablo Aparco para obtener el grado de Magister en Nutrición Pública por la Escuela de Posgrado de la Universidad Nacional Agraria La Molina.

Recibido: 13/11/2018 Aprobado: 06/03/2019 En línea: 20/03/2019
}

Citar como: Aparco JP, Bullón L, Cusirramos S. Impacto de micronutrientes en polvo sobre la anemia en niños de 10 a 35 meses de edad en Apurímac, Perú. Rev Peru Med Exp Salud Pública. 2019;36(1):17-25. doi:10.17843/rpmesp.2019.361.4042. 


\section{INTRODUCCIÓN}

En el Perú la anemia persiste como un problema severo de salud pública. En el 2018 afectó al 43,6\% de los niños menores de tres años y la tendencia de la anemia, a nivel nacional, entre 2012 a 2018 muestra un estancamiento de la reducción sin cambios significativos en este periodo ${ }^{(1,2)}$.

La anemia tiene consecuencias durante toda la vida, afectando la salud, educación y rendimiento laboral; estos efectos se traducen en costos para la familia y la sociedad ${ }^{(3-5)}$. Además, se ha estimado que la anemia ocasionó al Perú una pérdida aproximada de S/ 2777 millones para el 2011 (el 0,62\% del producto bruto interno de ese año), siendo mayor el costo para la economía rural que para la urbana ${ }^{(6)}$.

En 2010, el Perú inició la intervención piloto con micronutrientes en polvo (MNP) en tres regiones del país: Apurímac, Ayacucho y Huancavelica. Se eligió este suplemento porque la evidencia mostraba mayor aceptabilidad frente al sulfato ferroso ${ }^{(7,8)}$. A la fecha, se han publicado dos estudios sobre la efectividad de esta intervención piloto, con resultados diferentes, el primer estudio no encontró efectividad de los MNP para reducir la anemia al comparar las prevalencias regionales

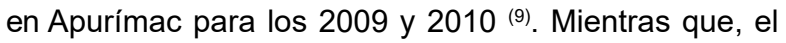
segundo estudio, con un diseño de vigilancia centinela en 29 establecimientos de salud del Ministerio de Salud (MINSA) de las tres regiones, reportó una reducción de 37 puntos porcentuales en la prevalencia de la anemia ${ }^{(10)}$; sin embargo, la estimación del impacto podría tener algunos sesgos, debido a la pérdida de la muestra ${ }^{(11)}(42 \%$ del total) y por no contar con grupo control ${ }^{(12)}$.

Para evaluar el impacto de una intervención sanitaria sin grupo control se pueden aplicar diversas técnicas multivariadas para generar controles estadísticos como el propensity score matching (PSM) ${ }^{(13,14)}$ que permite identificar controles (con características lo más similares posibles) para cada sujeto que recibe tratamiento, emparejando individuos de cada grupo según la probabilidad de participación dadas sus covariables ${ }^{(15)}$. Esta técnica estima el «efecto del tratamiento en tratados» (Average Treatment Treated, en inglés), es decir el impacto entre los que cumplieron el tratamiento, una subpoblación del total ${ }^{(14,16)}$. EI PSM se ha usado en el Perú para evaluar efectos nutricionales en programas como JUNTOS ${ }^{(17-19)}$ y el Vaso de Leche $(P V L){ }^{(20)}$.

Frente a este panorama de evidencia contradictoria del efecto de los MNP en nuestro país, se llevó a cabo el presente estudio con el objetivo de evaluar el impacto de la suplementación con MNP en la reducción de anemia en niños de 10 a 35 meses de edad de Apurímac, generando un grupo de control a través del PSM para reducir los sesgos en la estimación del efecto.

\section{MENSAJES CLAVE}

Motivación para realizar el estudio. En nuestro país, los estudios del efecto de los micronutrientes en polvo (MNP) sobre la anemia muestran resultados contradictorios, por lo que es necesario generar más evidencia sobre el impacto de los MNP.

Principales hallazgos. La prevalencia de anemia fue significativamente menor en los niños que consumieron 60 o más sobres de MNP comparados con sus controles; asimismo, la concentración de hemoglobina fue mayor en el grupo de intervención.

Implicancias. Se debe asegurar una provisión oportuna y completa de MNP a los niños menores de tres años y monitorear el consumo del suplemento, pues los efectos sobre la anemia se encuentran en niños que reciben y consumen el MNP.

\section{MATERIALES Y MÉTODOS}

\section{DISEÑO DE ESTUDIO}

Realizamos un análisis secundario de la base de datos de la Evaluación de la suplementación con MNP en niños de 6 a 35 meses de edad de Apurímac, ejecutada por el Centro Nacional de Alimentación y Nutrición (CENAN) ${ }^{(9)}$. El diseño del estudio original comprendía dos evaluaciones transversales; la evaluación basal en 2009 (sin distribución de MNP) y la final en 2010 (con distribución de MNP), para el estudio usamos la información de las dos evaluaciones.

\section{POBLACIÓN DE ESTUDIO Y MUESTRA}

La muestra original de la evaluación basal y final fue probabilística, multietápica e independiente por área urbana y rural de la región Apurímac. Se aplicó una encuesta para recolectar información socioeconómica, de salud, alimentación y dosaje de hemoglobina a 780 niños de 6 a 35 meses de edad en la evaluación basal; mientras que en la final se recogieron los mismos datos en 714 niños de la misma edad.

En el estudio incluimos para el grupo de control niños de ambos sexos con edades entre 10 a 35 meses de edad y que no consumieron ningún (cero) sobre de MNP; mientras que para el grupo de intervención se incluyeron niños de ambos sexos con edades entre 10 a 35 meses de edad que consumieron 60 o más sobres de MNP en un período de seis meses. La muestra final para el presente estudio fue de 1163 niños, de ellos 442 fueron tratamiento y 721 potenciales controles.

\section{MICRONUTRIENTES EN POLVO}

Los detalles de la formulación del suplemento, la dosis, la forma, frecuencia y canal de entrega del MNP, así como la duración de la intervención y la cuantificación de sobres consumidos han sido descritos en un estudio previo ${ }^{(9)}$. 


\section{VARIABLES}

Las variables de estudio también se han detallado en el estudio de Huaman-Espino et al. (9), aquí describimos brevemente que la variable independiente (intervención) se categorizó en dos: 1) tratamiento: niños con consumo de 60 o más sobres de MNP; y 2) control: niños que no consumieron ningún (cero) sobre de MNP. Las variables de respuesta (dependientes) fueron la prevalencia de anemia, definida por valores menores de $110 \mathrm{~g} / \mathrm{L}$ de hemoglobina ajustada por altitud ${ }^{(9)}$ y el nivel promedio de hemoglobina en sangre. Las variables de emparejamiento para estimar la probabilidad de participación en el programa fueron las características del niño, de la madre, familia, vivienda y localidad (ver Tabla 1).

\section{PROCESAMIENTO DE DATOS}

Fusionamos las dos bases de datos en una base única, luego seleccionamos niños que tuvieran entre 10 y 35 meses de edad, incluimos niños con al menos diez meses de edad para que tuvieran oportunidad de completar el consumo 60 sobres de MNP. Los registros fuera del rango de edad seleccionado fueron eliminados. A continuación, identificamos niños sin consumo de MNP (cero sobres) y con consumo de 60 o más sobres de MNP y retiramos los datos de niños con consumo de MNP entre 1 a 59 sobres. También recategorizamos las variables edad del niño (tres categorías), nivel educativo de la madre (tres categorías) y altitud (cuatro categorías) y en cada una de las categorías creamos variables indicadoras binarias («ficticias») para flexibilizar el modelo de estimación de la probabilidad de participación como recomiendan Guo y Fraser ${ }^{(21)}$.

\section{ANÁLISIS DE DATOS}

Finalizado el procesamiento de la base de datos y contando sólo con niños de 10 a 35 meses de edad y que cumplían los criterios de inclusión sobre el consumo de MNP estimamos el impacto a través del PSM, aplicando los siguientes procedimientos:

a. Estimación de la probabilidad de participación en la intervención: para el cálculo de la probabilidad de cada niño de participar en la intervención (consumir 60 o más sobres de MNP) empleamos un modelo probit con las variables sociodemográficas del niño, la madre y las características de la vivienda. Inicialmente se incluyeron todas las variables de la Tabla 1 y luego seleccionamos el modelo probit que cumplió con: i) tener observaciones en cada bloque de datos ( $\sin$ vacíos); ii) tener muestras equilibradas, es decir que las variables observables estuvieran balanceadas (balancing property) en cada bloque de ambos grupos de estudio; y iii) que perdiera la menor cantidad de datos en el emparejamiento ${ }^{(14,21)}$. Luego de seleccionar el modelo probit estimamos el puntaje de propensión de cada individuo en ambos grupos de estudio. b. Restricción de la muestra al área de soporte común: para garantizar observaciones dentro del área de soporte común, ordenamos las probabilidades de participación de menor a mayor y aplicamos el criterio de observaciones mínimas y máximas lo que nos permitió eliminar observaciones fuera del rango común.

c. Emparejamiento de los grupos de estudio: se aplicaron tres algoritmos de emparejamiento, para verificar la variabilidad de los resultados: i) Estimador de PSM por vecino más cercano con cinco controles y restricción en la distancia máxima de 0,08 , en la cual se toma a cada individuo del grupo de tratamiento y se buscan cinco controles con la probabilidad de participación más cercana, es decir los vecinos más cercanos; ii) Estimador de PSM por kernel, es un estimador no paramétrico que empareja a cada individuo del tratamiento con un promedio ponderado de (potencialmente) todos los individuos del control, aplicando los pesos kernel; y iii) Estimador de PSM por regresión lineal local, es la regresión de la variable objetivo del grupo control en el vecindario de cada observación del grupo tratado sobre un intercepto y se le adiciona un término de pendiente, que es la probabilidad de participación (11).

d. Estimación del impacto de la intervención: determinamos el impacto de la intervención aplicando el comando "psmatch2" del programa Stata, que estima la media ponderada de diferencia entre el grupo de intervención y grupo de control, utilizando como función de peso el valor del PSM obtenido de los participantes. El impacto estimado corresponde al efecto del tratamiento en los tratados (ATT), es decir el efecto en niños que consumieron 60 o más sobres de MNP, comparados con los niños control $(14,16)$.

e. Estimación de errores estándar e intervalos de confianza: se calcularon los errores estándar y los intervalos de confianza usando "bootstrapping" con 150 repeticiones.

Para las estimaciones usamos el programa Stata v14 SE v14.2 (Stata Corporation, College Station, Texas, USA) y en todos los casos consideramos los intervalos de confianza al $95 \%$ (IC 95\%) y el valor $p<0,05$ y $p<0,01$ como nivel de significancia estadística.

\section{ASPECTOS ÉTICOS}

La evaluación de la intervención con MNP en Apurímac del CENAN fue aprobada por el Comité de Ética del Instituto Nacional de Salud con registro Ol-049-10. Además, se obtuvo la autorización de la Dirección General del CENAN para el uso de las bases de datos.

\section{RESULTADOS}

Se analizaron los datos 1163 niños de 10 a 35 meses de edad, 442 del grupo de tratamiento y 721 del grupo control. 
Tabla 1. Variables de estudio y emparejamiento.

\begin{tabular}{|c|c|}
\hline Variable & Descripción \\
\hline \multicolumn{2}{|l|}{ Variable independiente } \\
\hline Tratamiento & $\begin{array}{l}\text { Describe la intervención con MNP. Toma dos valores: 1: si el niño consumió } 60 \text { o más sobres de MNP y 0: si el niño no } \\
\text { consumió ningún sobre. }\end{array}$ \\
\hline \multicolumn{2}{|l|}{ Variables dependientes } \\
\hline Anemia & $\begin{array}{l}\text { Define la presencia de la enfermedad en el niño: Toma dos valores: } 1 \text { : si el niño tiene menos de } 110 \mathrm{~g} / \mathrm{L} \text { de hemoglobina } \\
\text { ajustada por altitud }{ }^{(9)} \text {; y } 0 \text { : si el niño tiene } 110 \mathrm{~g} / \mathrm{L} \text { de hemoglobina o más. }\end{array}$ \\
\hline Hemoglobina & Concentración de hemoglobina ( $\mathrm{g} / \mathrm{dL}$ ) en sangre. \\
\hline \multicolumn{2}{|l|}{ Variables de control } \\
\hline Sexo del niño (a) & 1: varón; y 0: mujer. \\
\hline Niños de 10 a 18 meses & 1: niño de 10 a 18 meses; y 0: otro valor. \\
\hline Niños de 19 a 24 meses & 1: niño de 19 a 24 meses; y 0: otro valor. \\
\hline Niños de 25 a 35 meses & 1: niño de 25 a 35 meses: $y$ 0: otro valor. \\
\hline Beneficiario de programa social & 1: niño es beneficiario de JUNTOS, Vaso de Leche o Cunamás; y 0: si no es beneficiario de ningún programa social. \\
\hline Edad de la madre & Describe la edad de las madres en años. \\
\hline Idioma de la madre español & 1: si la madre habla predominantemente en quechua; y 0: otra opción. \\
\hline Idioma de la madre quechua & 1: si la madre habla predominantemente en español; y 0: otra opción. \\
\hline $\begin{array}{l}\text { Idioma de la madre quechua y } \\
\text { español }\end{array}$ & 1: si la madre si la madre se comunica con facilidad en ambos idiomas; y 0: otra opción. \\
\hline Madre sin educación formal & 1: si la madre no tiene instrucción o no ha aprobado ningún grado en primaria; y 0: otra opción. \\
\hline Madre con educación primaria & 1: si la madre ha aprobado como mínimo el primer grado de primaria o hasta el sexto grado de primaria; y 0: otra opción. \\
\hline $\begin{array}{l}\text { Madre con educación secundaria } \\
\text { o más }\end{array}$ & 1: si la madre ha aprobado como mínimo el primer grado de secundaria o hasta el nivel superior; y 0: otra opción. \\
\hline Madre trabaja & $\begin{array}{l}\text { 1: si la madre se encontraba al día de la encuesta trabajando o de vacaciones; y 0: si la madre al día de la encuesta no } \\
\text { tenía trabajo. }\end{array}$ \\
\hline Número de miembros en el hogar & Describe el número de miembros en el hogar. \\
\hline $\begin{array}{l}\text { Material adecuado de las } \\
\text { paredes }\end{array}$ & $\begin{array}{l}\text { 1: adecuado si la pared de la vivienda es predominantemente de ladrillo, piedra, adobe, quincha o madera; y 0: inadecuado } \\
\text { si la pared es de triplay, estera, cartón u otro. }\end{array}$ \\
\hline Material adecuado del techo & $\begin{array}{l}\text { 1: adecuado si el techo de la vivienda es predominantemente de concreto, madera, tejas o plancha de calamina; y 0: } \\
\text { inadecuado si el techo es de estera, cañas, paja, hojas de palmera, plástico o cartón. }\end{array}$ \\
\hline Material adecuado del piso & $\begin{array}{l}\text { 1: adecuado si el piso de la vivienda es predominantemente de parquet/madera, lamina asfáltica/vinílico, losetas/terrazos } \\
\text { o cemento pulido; y 0: inadecuado si el piso es de tierra o arena. }\end{array}$ \\
\hline Acceso adecuado a agua segura & $\begin{array}{l}\text { 1: acceso adecuado a agua segura si la fuente de agua es de red pública dentro o fuera de la vivienda; y 0: acceso } \\
\text { inadecuado si la fuente es pozo en casa o público, agua de superficie, rio, lluvia o camión tanque. }\end{array}$ \\
\hline $\begin{array}{l}\text { Disposición adecuada de } \\
\text { excretas }\end{array}$ & $\begin{array}{l}\text { 1: disposición adecuada de excretas si el servicio higiénico está conectado a red pública dentro o fuera de la vivienda o } \\
\text { es letrina bajo pautas técnicas; y } 0 \text { : disposición inadecuada si el servicio higiénico es una letrina que no cumple pautas } \\
\text { técnicas, hacen sus deposiciones en un pozo ciego, en el campo, en el rio o acequia. }\end{array}$ \\
\hline Acceso a electricidad & 1: si la vivienda tiene electricidad; y 0 : si la vivienda no cuenta con electricidad. \\
\hline Uso de combustible adecuado & $\begin{array}{l}\text { 1: adecuado si en la vivienda usan como combustible para cocinar, electricidad o gas; y 0: inadecuado si la vivienda usa } \\
\text { carbón, leña o bosta. }\end{array}$ \\
\hline Hacinamiento en el hogar & 1: vivienda con hacinamiento si hay más de tres personas por habitación; y 0: sin hacinamiento la situación contraria. \\
\hline Tenencia de radio en el hogar & 1: tiene si algún miembro del hogar posee una radio; y 0: no tiene en la situación contraria. \\
\hline Tenencia de televisor & 1: tiene si algún miembro del hogar posee un televisor que funciona; y 0: no tiene en la situación contraria. \\
\hline Tenencia de computadora & 1: tiene si algún miembro del hogar posee una computadora que funciona, y 0: no tiene en la situación contraria. \\
\hline Tenencia de refrigeradora & 1: tiene si el hogar posee una refrigeradora que funciona; y 0: no tiene en la situación contraria. \\
\hline Tenencia de teléfono & 1: tiene si algún miembro del hogar tiene teléfono sea fijo o celular que funcione; y 0: no tiene en la situación contraria. \\
\hline Área rural & $\begin{array}{l}\text { 1: rural si la vivienda del niño se encuentra en un centro poblado que no tiene más de } 100 \text { viviendas agrupadas } \\
\text { contiguamente ni es capital de distrito; o que teniendo más de } 100 \text { viviendas, éstas se encuentran dispersas o diseminadas } \\
\text { sin formar bloques o núcleos; y 0: urbana cuando la vivienda se encuentra en un centro poblado que tiene como mínimo } \\
100 \text { viviendas agrupadas contiguamente (en promedio } 500 \text { habitantes). Por excepción se incluyen a todos los centros } \\
\text { poblados capitales de distrito, aun cuando no reúnan la condición indicada. }\end{array}$ \\
\hline Altitud menos de $2500 \mathrm{~m} \mathrm{s.} \mathrm{n.} \mathrm{m.}$ & 1: si la vivienda del niño está a una altitud de menos de $2500 \mathrm{~m} \mathrm{s.} \mathrm{n.} \mathrm{m.;} \mathrm{y} \mathrm{0:} \mathrm{cualquier} \mathrm{otro} \mathrm{valor.}$ \\
\hline Altitud de 2500 a $31199 \mathrm{~m}$ s. n. m. & 1: si la vivienda del niño está a una altitud de entre 2500 a 3199 m s. n. m.; y 0: cualquier otro valor. \\
\hline Altitud de 3200 a 3699 m s. n. m. & 1: si la vivienda del niño está a una altitud de entre 3200 a 3699 m s. n. m.; y 0: cualquier otro valor. \\
\hline Altitud más de 3700 m s. n. m. & 1: si la vivienda del niño está a una altitud de más de 3700 m s. n. m.; y 0: cualquier otro valor. \\
\hline
\end{tabular}

m.s.n.m: metros sobre el nivel del mar 


\section{ESTIMACIÓN DE LA PROBABILIDAD DE PARTICIPACIÓN (PROPENSITY SCORE)}

La probabilidad de participar en la intervención con MNP se reducía para los niños que tenían viviendas con paredes de material adecuado, vivían a mayor altitud y en el área rural; mientras que aumentaba en los niños que tenían viviendas sin acceso a electricidad, con hacinamiento, radio y cuyas madres tenían nivel educativo secundario o más (Tabla 2).

\section{COMPARACIÓN DE LOS GRUPOS ANTES Y DESPUÉS DEL EMPAREJAMIENTO}

En la Tabla 3 se comparan las características del grupo de tratados y control antes y después del emparejamiento; al respecto encontramos que inicialmente las variables de la madre como idioma quechua y del hogar como material de pared, acceso a electricidad, a combustible adecuado, hacinamiento, tenencia de radio, tenencia de teléfono y altitud mayor a $3700 \mathrm{~m} \mathrm{s.} \mathrm{n.} \mathrm{m.,} \mathrm{mostraban} \mathrm{diferencias}$ significativas entre grupos, pero luego del emparejamiento no existieron diferencias.

\section{ESTIMACIÓN DEL IMPACTO}

El impacto del MNP sobre la anemia en el grupo de intervención, al aplicar el modelo vecino más cercano con cinco controles, fue de $-11,4$ puntos porcentuales (tratados: $36,0 \%$ y controles: $47,4 \%$ ); asimismo al aplicar los algoritmos de PSM, kernely regresión lineal local encontramos efectos similares (reducción de 10,8 puntos porcentuales y 11,3 puntos porcentuales respectivamente). Para estimar los errores estándar y los intervalos de confianza se aplicó la técnica de bootstrap y se encontró que en todos los casos la reducción resultó estadísticamente significativa (Tabla 4). También estimamos el efecto del MNP sobre los niveles de hemoglobina y observamos que en el modelo vecino más cercano la diferencia fue de $0,33 \mathrm{~g} / \mathrm{dL}$, siendo mayor en el grupo de intervención comparado con los controles $(11,22$ y 10,89 respectivamente). Las diferencias fueron similares (0,29 y 0,31 respectivamente) al estimar el impacto con los otros dos algoritmos de emparejamiento y en los tres casos las diferencias fueron significativas (Tabla 5).

Tabla 2. Estimación de la probabilidad de consumir 60 o más sobres de micronutrientes en polvo.

\begin{tabular}{|c|c|c|c|}
\hline \multirow{2}{*}{ Variable } & \multicolumn{3}{|c|}{ Consumo de 60 o más sobres de MNP } \\
\hline & Efectos marginales & IC $95 \%$ & Valor de $p$ \\
\hline Sexo & $-0,013$ & $-0,070-0,043$ & 0,640 \\
\hline Niños de 19 a 24 meses & 0,088 & $0,004-0,172$ & 0,038 \\
\hline Niños de 25 a 35 meses & 0,047 & $-0,018-0,112$ & 0,155 \\
\hline Beneficiario de programa social & $-0,092$ & $-0,190-0,006$ & 0,081 \\
\hline Edad de la madre (años) & $-0,001$ & $-0,005-0,003$ & 0,742 \\
\hline Idioma de la madre quechua & $-0,015$ & $-0,102-0,073$ & 0,740 \\
\hline Idioma de la madre quechua y español & 0,005 & $-0,654-0,075$ & 0,899 \\
\hline Madre con educación primaria & $-0,012$ & $-0,129-0,105$ & 0,838 \\
\hline Madre con educación secundaria o más & 0,135 & $0,021-0,249$ & 0,023 \\
\hline Madre trabaja & $-0,025$ & $-0,090-0,040$ & 0,451 \\
\hline Material adecuado de las paredes & $-0,084$ & $-0,169-0,001$ & 0,049 \\
\hline Material adecuado del techo & 0,029 & $-0,074-0,133$ & 0,575 \\
\hline Material adecuado del piso & 0,007 & $-0,077-0,091$ & 0,868 \\
\hline Acceso adecuado a agua segura & $-0,017$ & $-0,105-0,070$ & 0,699 \\
\hline Disposición adecuada de excretas & $-0,020$ & $-0,089-0,049$ & 0,570 \\
\hline Acceso a electricidad & 0,088 & $0,009-0,168$ & 0,035 \\
\hline Uso de combustible adecuado & $-0,064$ & $-0,145-0,017$ & 0,119 \\
\hline Hacinamiento en el hogar & 0,080 & $0,021-0,139$ & 0,008 \\
\hline Tenencia de radio en el hogar & 0,096 & $0,008-0,184$ & 0,043 \\
\hline Tenencia de teléfono & 0,062 & $-0,010-0,134$ & 0,089 \\
\hline Área rural & $-0,117$ & $-0,198--0,036$ & 0,005 \\
\hline Altitud 2500 a 3199 m s. n. m. & $-0,213$ & $-0,509-0,082$ & 0,171 \\
\hline Altitud de 3200 a 3699 m s. n. m. & $-0,235$ & $-0,531-0,062$ & 0,132 \\
\hline Altitud más de 3700 m s. n. m. & $-0,273$ & $-0,459--0,088$ & 0,040 \\
\hline
\end{tabular}

Nota: En la regresión probit, la variable dependiente equivale a 1 si el niño consumió 60 o más sobres de micronutrientes en polvo y 0 si no consumió ningún sobre de micronutrientes en polvo. Los efectos marginales representan la contribución de cada variable a la probabilidad de que un niño consuma 60 o más sobres de micronutrientes en polvo.

MNP: Micronutriente en polvo, m.s.n.m: metros sobre el nivel del mar. 


\section{DISCUSIÓN}

Los resultados del estudio muestran que la intervención con MNP tuvo impacto para reducir la prevalencia de anemia y mejorar la concentración de hemoglobina en niños de 10 a 35 meses de edad que consumieron 60 o más sobres de MNP (tratados) comparados con sus controles. Estos resultados son concordantes con estudios en diversos países que han reportado eficacia y efectividad de los MNP para reducir la anemia y mejorar la concentración de los marcadores biológicos de hierro como hemoglobina, ferritina y receptores de transferrina entre otros ${ }^{(8,22)}$.

En nuestro país los resultados difieren con lo reportado por Huaman-Espino et al., que no encontró impacto del MNP sobre la anemia ${ }^{\left({ }^{9}\right)}$, esta discrepancia se explicaría por las diferencias en los diseños de los estudios. Huaman-Espino et al. realizó una evaluación poblacional incluyendo a todos los niños sin considerar si habían consumido la dosis mínima de 60 sobres de MNP (23); mientras que en nuestro estudio estimamos el efecto del tratamiento en tratados es decir seleccionamos a los niños que consumieron 60 sobres de MNP o más y los comparamos con sus controles estadísticos (niños sin consumo de MNP). Estos resultados diferentes evidencian que la adherencia es un requisito indispensable para esperar impacto de las intervenciones con hierro; además Huaman-Espino et al. reportó que la baja adherencia no sólo estaba condicionada al consumo del niño sino también a la distribución incompleta, discontinua y sin orientación del uso del MNP ${ }^{(9)}$.

Por otra parte, nuestros hallazgos coinciden con lo reportado por Munayco et al., que encontraron impacto del MNP para reducir la prevalencia de anemia, aunque la magnitud de la reducción difiere en casi cinco veces ${ }^{\left({ }^{10}\right)}$. Las razones de las diferencias entre los dos estudios podrían deberse a que el estudio de Munayco et al. utilizó mayores dosis de suplementación (hasta 180 sobres de MNP) y los diseños de evaluación empleados fueron diferentes, pues los autores desarrollaron una vigilancia centinela sin grupo de control y al finalizar el seguimiento reportaron una pérdida de $42,7 \%$ de la muestra inicial. Estas características del estudio podrían introducir algunos sesgos en la determinación del tamaño del efecto como el de pérdida de muestra ${ }^{(11)}$ y maduración biológica ${ }^{(12)}$. En nuestro caso aplicamos la evaluación con datos transversales de dos años consecutivos en la misma región y generamos un grupo de control a través del PSM, para reducir el sesgo de maduración biológica por lo que consideramos que nuestras estimaciones podrían ser más precisas.

Tabla 3. Comparación del grupo de tratamiento y control antes y después del emparejamiento.

\begin{tabular}{|c|c|c|c|c|c|c|}
\hline \multirow[b]{2}{*}{ Variables } & \multicolumn{3}{|c|}{ Sin emparejamiento } & \multicolumn{3}{|c|}{ Con emparejamiento } \\
\hline & $\begin{array}{c}\text { Tratados } \\
\text { (\%) }\end{array}$ & $\begin{array}{c}\text { Potenciales } \\
\text { controles } \\
(\%)\end{array}$ & $\begin{array}{l}\text { Valor } \\
\text { de } p\end{array}$ & $\begin{array}{c}\text { Tratados } \\
(\%)\end{array}$ & $\begin{array}{c}\text { Controles } \\
\text { emparejados } \\
(\%)\end{array}$ & $\begin{array}{l}\text { Valor } \\
\text { de } p\end{array}$ \\
\hline Sexo & 47,9 & 50,0 & 0,486 & 48,6 & 49,9 & 0,699 \\
\hline Niños de 19 a 24 meses & 21,4 & 17,6 & 0,102 & 21,3 & 17,7 & 0,180 \\
\hline Niños de 25 a 35 meses & 47,2 & 46,0 & 0,682 & 47,0 & 46,1 & 0,806 \\
\hline Beneficiario de programa social & 85,9 & 95,7 & 0,578 & 87,1 & 94,7 & 0,697 \\
\hline Edad de la madre (años) & 29,1 & 29,3 & 0,631 & 29,1 & 29,3 & 0,695 \\
\hline Idioma de la madre quechua & 22,8 & 28,2 & 0,041 & 22,9 & 28,0 & 0,182 \\
\hline Idioma de la madre quechua y español & 47,9 & 46,1 & 0,556 & 48,1 & 46,2 & 0,575 \\
\hline Madre con educación primaria & 25,5 & 37,0 & $<0,001$ & 26,1 & 36,5 & 0,001 \\
\hline Madre con educación secundaria o más & 68,1 & 53,3 & $<0,001$ & 67,6 & 53,1 & $<0,001$ \\
\hline Madre trabaja & 65,1 & 67,5 & 0,403 & 65,5 & 67,4 & 0,560 \\
\hline Material adecuado de las paredes & 80,3 & 85,5 & 0,019 & 80,9 & 85,4 & 0,078 \\
\hline Material adecuado del techo & 12,2 & 14,9 & 0,187 & 12,3 & 14,8 & 0,287 \\
\hline Material adecuado del piso & 74,4 & 77,2 & 0,274 & 74,7 & 77,0 & 0,423 \\
\hline Acceso adecuado a agua segura & 18,3 & 22,7 & 0,073 & 18,9 & 22,2 & 0,154 \\
\hline Disposición adecuada de excretas & 43,2 & 46,0 & 0,346 & 43,9 & 45,8 & 0,496 \\
\hline Acceso a electricidad & 80,9 & 74,2 & 0,008 & 80,3 & 76,4 & 0,072 \\
\hline Uso de combustible adecuado & 64,9 & 70,8 & 0,034 & 65,7 & 69,2 & 0,103 \\
\hline Hacinamiento en el hogar & 52,9 & 46,0 & 0,022 & 51,5 & 47,9 & 0,082 \\
\hline Tenencia de radio en el hogar & 91,4 & 87,7 & 0,045 & 90,0 & 89,1 & 0,114 \\
\hline Tenencia de teléfono & 38,4 & 31,6 & 0,017 & 36,2 & 34,4 & 0,183 \\
\hline Área rural & 40,4 & 41,1 & 0,815 & 40,3 & 41,2 & 0,784 \\
\hline Altitud 2500 a 3199 m.s.n.m & 46,8 & 45,0 & 0,560 & 46,7 & 45,2 & 0,653 \\
\hline Altitud de 3200 a 3699 m.s.n.m & 49,0 & 49,3 & 0,926 & 49,5 & 49,3 & 0,954 \\
\hline Altitud más de 3700 m.s.n.m & 2,7 & 4,9 & 0,048 & 2,9 & 4,6 & 0,106 \\
\hline
\end{tabular}

m.s.n.m: metros sobre el nivel del mar. 
Tabla 4. Impacto de la suplementación con micronutrientes en polvo sobre la prevalencia de anemia.

\begin{tabular}{lcccccccc}
\hline $\begin{array}{l}\text { Tipo de emparejamiento } \\
\text { (matching) }\end{array}$ & $\begin{array}{c}\text { Tratados } \\
\mathbf{( \% )}\end{array}$ & $\begin{array}{c}\text { Control } \\
\mathbf{( \% )}\end{array}$ & $\begin{array}{c}\text { ATT } \\
\text { (Tratados - } \\
\text { Control) }\end{array}$ & Prueba t & $\begin{array}{c}\text { Error } \\
\text { estándar }\end{array}$ & $\mathbf{z}$ & $\begin{array}{c}\text { Valor } \\
\text { de } \mathbf{p}\end{array}$ & IC 95\% \\
\hline Vecino más cercano (NN) & 36,0 & 47,4 & $-11,4$ & $-3,39$ & 0,036 & $-3,28$ & 0,001 & $-0,190--0,048$ \\
Kernel & 36,0 & 46,9 & $-10,9$ & $-3,53$ & 0,263 & $-4,17$ & $<0,001$ & $-0,161--0,058$ \\
Regresión lineal local & 36,2 & 47,5 & $-11,3$ & $-2,71$ & 0,327 & $-3,46$ & 0,001 & $-0,177--0,049$ \\
\hline
\end{tabular}

ATT: efecto promedio entre los tratados (average treatment effect on treated).

La técnica del PSM es empleada para estimar impacto cuando las intervenciones no tienen una regla clara de asignación, no son aleatorizadas, son de cobertura universal, no tienen grupo de control o la distribución de características observables es diferente en los grupos de comparación ${ }^{(14,16,21)}$. En el campo de la salud, varios estudios han empleado la técnica del PSM para estimar el impacto de las diversas intervenciones, así en México, Neufeld et al., empleando el PSM, evaluaron el impacto del Programa Oportunidades y reportaron efectos significativos en la reducción de anemia en niños de 6 a 24 meses ${ }^{(24)}$ (en mayores de 24 meses no encontró impacto), lo cual concuerda con nuestros resultados. Por su parte Leroy et al., aplicaron el PSM con diferencias en diferencias para determinar el efecto del Programa Oportunidades sobre la talla, el peso para edad y variación del peso, encontrando efecto en los tres indicadores sólo para los niños que iniciaron el programa desde los seis meses de edad. ${ }^{(25)}$. Gutiérrez et al., también estimaron el impacto de Oportunidades sobre la morbilidad infantil, aplicando PSM y hallaron efecto significativo reduciendo el número de días de los episodios de las enfermedades infantiles ${ }^{(26)}$.

En el Perú también se ha aplicado el PSM para evaluar el impacto de algunas intervenciones nutricionales. Uno de los programas sociales que por sus características de asignación ha empleado el PSM es JUNTOS, desarrollando hasta tres evaluaciones con esta técnica, la primera realizada en 2010 , los autores utilizan el puntaje de propensión a nivel de distrito y hogar, y reportan que los resultados no muestran impacto en indicadores finales como talla o niveles de hemoglobina, pero si efectos favorables en utilización de servicios de salud y mejora de gasto en alimentos más nutritivos ${ }^{(17)}$.

El estudio tiene limitaciones propias del diseño y de la técnica de PSM empleada, en primer término, la cuantificación de sobres de MNP consumidos, en el estudio original, podría haberse afectado con los sesgos de memoria y deseabilidad social; sin embargo, en la intervención piloto, se acondicionaron los carnés de Crecimiento y Desarrollo (CRED) para registrar la recepción y consumo de MNP y reducir el olvido; esto se evidenciaría en la baja proporción de niños que recibieron al menos 60 sobres $(49 \%)^{(9)}$.

Otro aspecto a resaltar entre las limitaciones de nuestro estudio inherentes al uso del PSM, es que evaluamos el impacto en niños de 10 a 35 meses por lo que el resultado no sería aplicable a niños menores; al respecto debemos mencionar que la Iniciativa Global de Salud Sprinkles recomienda evaluar el efecto del MNP en niños que han tenido un periodo mínimo de exposición a los suplementos ${ }^{(27)}$, en nuestro caso por las limitaciones en la distribución consideramos que a los 10 meses de edad (4 meses de exposición) el niño tendría oportunidad de recibir y consumir como mínimo 60 sobres para que el efecto sobre la anemia sea plausible. Otra limitación de la técnica de PSM es que estima el impacto en una subpoblación de los niños asignados a tratamiento, el subgrupo de niños que recibieron y consumieron como mínimo 60 sobres, este aspecto limitaría su aplicación universal o poblacional; sin embargo, al generar un grupo de control en la misma región y edad, contar con una gran base de datos y aplicar varios algoritmos para la estimación del impacto, los resultados podrían estar cercanos al impacto poblacional ${ }^{(14)}$.

El estudio tiene como fortalezas usar una base de datos con 1494 niños, proveniente de dos evaluaciones transversales en la misma población en dos años consecutivos y tener una pérdida menor de $1 \%$ de datos. Además el cumplimiento de los supuestos para aplicar PSM como independencia condicional, existencia de suficientes variables de emparejamiento y un área de soporte común amplia ${ }^{(28)}$ lo cual se evidencia en la baja perdida de datos (siete niños). Finalmente constituye otra fortaleza que los resultados del

Tabla 5. Impacto de la suplementación con micronutrientes en polvo sobre el promedio de hemoglobina.

\begin{tabular}{lcccccccc}
\hline $\begin{array}{l}\text { Tipo de emparejamiento } \\
\text { (matching) }\end{array}$ & $\begin{array}{c}\text { Tratados } \\
\mathbf{( X )}\end{array}$ & $\begin{array}{c}\text { Control } \\
\mathbf{( X )}\end{array}$ & $\begin{array}{c}\text { ATT } \\
\text { (Tratados - } \\
\text { Control) }\end{array}$ & Prueba t & $\begin{array}{c}\text { Error } \\
\text { estándar }\end{array}$ & $\mathbf{z}$ & $\begin{array}{c}\text { Valor } \\
\text { de } \mathbf{p}\end{array}$ & IC 95\% \\
\hline Vecino más cercano (NN) & 11,22 & 10,89 & 0,33 & 4,05 & 0,096 & 3,51 & $<0,001$ & $0,148-0,523$ \\
\hline Kernel & 11,22 & 10,93 & 0,29 & 3,91 & 0,792 & 3,69 & $<0,001$ & $0,137-0,447$ \\
Regresión lineal local & 11,22 & 10,90 & 0,31 & 2,97 & 0,073 & 4,34 & $<0,001$ & $0,175-0,462$ \\
\hline
\end{tabular}

ATT: efecto promedio entre los tratados (average treatment effect on treated). 
impacto fueron concordantes al aplicar los tres algoritmos de emparejamiento y que para la estimación de intervalos de confianza se aplicó la técnica de bootstrap que permite estimaciones robustas ${ }^{(14)}$.

La intervención con MNP tuvo impacto reduciendo la prevalencia de anemia hasta en 11 puntos porcentuales y aumentando la concentración de hemoglobina en 0,33 g/dL en los niños tratados (consumieron 60 o más sobres de MNP) comparados con sus controles. Se recomienda que los servicios de salud mejoren la cobertura y calidad de la entrega de micronutrientes en polvo para garantizar que más niños pueden consumir la dosis mínima de MNP para obtener resultados.

Contribuciones de autoría: JPA, LB y SC han participado en la concepción del artículo, su redacción y aprobación de la versión final. Además, JPA y LB participaron en el procesamiento y análisis de datos.

\section{Fuente de financiamiento: autofinanciado}

Conflicto de interés: los autores no refieren conflictos de interés en la publicación de este artículo.

\section{REFERENCIAS BIBLIOGRÁFICAS}

1. Instituto Nacional de Estadística e Informática. Encuesta Nacional de Demografía y Salud (ENDES) 2012, Informe Principal. Lima: INEI; 2013 [citado el 12 noviembre de 2018]. Disponible en: http://proyectos.inei.gob. pe/web/biblioineipub/bancopub/Est/ Lib1075/index.html.

2. Instituto Nacional de Estadística e Informática. Indicadores de Resultados de los Programas Presupuestales 2013-2018. Encuesta Demográfica y Salud Familiar (Resultados Preliminares) [Internet]. Lima: INEI; 2017 [citado el 30 octubre de 2018]. Disponible en: https://proyectos. inei.gob.pe/endes/2018/ppr/Indicadores_de_Resultados_de_los_Programas Presupuestales_ENDES_Primer_Semestre_2018.pdf.

3. Zimmermann MB, Hurrell RF. Nutritional iron deficiency. Lancet. 2007;370(9586):511-20. doi: https://doi. org/10.1016/S0140-6736(07)61235-5.

4. Camaschella C. Iron-deficiency anemia. N Engl J Med. 2015;372(19):1832-43. doi: 10.1056/NEJMra1401038.

5. Zavaleta N, Astete-Robilliard L. Efecto de la anemia en el desarrollo infantil: consecuencias a largo plazo. Rev Peru Med Exp Salud Publica. 2017;34(4):716-22. doi: 10.17843/rpmesp.2017.344.3251.

6. Alcázar L. Impacto económico de la anemia en el Perú [Internet]. Lima: GRADE; Acción contra el Hambre; 2012 [citado el 4 de noviembre de 2018]. Disponible en: http://www.grade.org.pe/upload/ publicaciones/archivo/download/pubs/ LIBROGRADE_ANEMIA.pdf

7. Organización Mundial de la Salud. Directriz: Uso de micronutrientes en polvo para la fortificación domiciliaria de los alimentos consumidos por lactantes y niños de 6 a 23 meses de edad [Internet]. Ginebra: Organización Mundial de la Salud; 2012 [citado el 3 de noviembre de 2018]. Disponible en: https:// apps.who.int/iris/bitstream/handle/10665/101016/9789243502045_spa. pdf;jsessionid=FC4F63EA9174DA9BD102C4FF550CC358? sequence $=1$

8. De-Regil LM, Suchdev PS, Vist GE, Walleser S, Peña-Rosas JP. Home fortification of foods with multiple micronutrient powders for health and nutrition in children under two years of age (Review). Evid Based Child Health. 2013;8(1):112-201. doi: 10.1002/ebch.1895.

9. Huamán-Espino L, Aparco JP, Nuñez-Robles E, Gonzáles E, Pillaca J, Mayta-Tristán P. Consumo de suplementos con multimicronutrientes chispitas y anemia en niños de 6 a 35 meses: estudio transversal en el contexto de una intervención. Rev Peru Med Exp Salud Pública. 2012;29(3):31423.

10. Munayco CV, Ulloa-Rea ME, MedinaOsis J, Lozano-Revollar CR, Tejada V, Castro-Salazar C, Munarriz-Villafuerte C, Bustos C, Arias L. Evaluación del impacto de los multimicronutrientes en polvo sobre la anemia infantil en tres regiones andinas del Perú. Rev Peru Med Exp Salud Publica. 2013;30(2):229-234.

11. Shadish WR, Cook TD, Campbell DT. Experimental and quasi-experimental designs for generalized causal inference. New York: Houghton Mifflin Company; 2002.

12. Szklo M, Nieto FJ. Epidemiology: Beyond the basics. 3rd ed. Burlington, Mass: Jones \& Bartlett Learning; 2014.

13. Wooldridge JM. Introductory econometrics: a modern approach. 5th ed. Mason. Ohio: South-Western Cengage Learning; 2012.

14. Bernal R, Peña X. Guía práctica de evaluación de impacto. Bogotá: Universidad de los Andes. Facultad de Economía, Centro de Estudios sobre Desarrollo Económico; 2011.

15. Pattanayak CW, Rubin DB, Zell ER. Propensity score methods for creating covariate balance in observational studies. Rev Esp Cardiol. 2011;64(10):897-903. doi: 10.1016/j.recesp.2011.06.008.

16. GertlerP, MartinezS, PremandP, Rawlings L, Vermeersch CM. Impact Evaluation in Practice. 2nd ed. Washington DC: InterAmerican Development Bank/World Bank; 2016.

17. Perova E, Vaquis R. Welfare impacts of the "JUNTOS" Program in Peru: Evidence from a non-experimental Evaluation". Washington DC: World Bank; 2010.

18. Sánchez A, Jaramillo M. Impacto del programa JUNTOS sobre la nutrición temprana [Internet]. Lima: GRADE. Ed. Arteta; 2012 [citado el 3 de noviembre de 2018]. Disponible en: http://www.grade. org.pe/upload/publicaciones/archivo/ download/pubs/ddt61.pdf

19. Escobal J, Benites S. Algunos impactos del programa JUNTOS en el bienestar de los niños: Evidencia basada en el estudio Niños del Milenio. Young Lives. Boletín de políticas públicas sobre infancia $\mathrm{N}^{\circ} 5$ [Internet]. Lima: GRADE; 2012. [citado el 30 de octubre de 2018]. Disponible en: http://www.grade.org. $\mathrm{pe} /$ publicaciones/1074-algunos-impactos-del-programa-juntos-en-el-bienestar-de-los-ninos-evidencia-basada-en-el-estudio-ninos-del-milenio/

20. Gajate G, Inurritegui M. El impacto de los programas alimentarios sobre el nivel de nutrición infantil: una aproximación a partir de la metodología del "Propensity Score Matching" [Internet]. Lima: CIES; 2002 [citado el 4 de diciembre de 2018]. Disponible en: https://www.mef.gob.pe/ contenidos/pol_econ/documentos/VaspLeche_GajateInurritegui.pdf 
21. Guo S, Fraser MW. Propensity score analysis: Statistical methods and applications. Thousand Oaks, CA: Sage; 2009.

22. Das JK, Salam RA, Kumar R, Bhutta ZA. Micronutrient fortification of food and its impact on woman and child health: a systematic review. Syst Rev. 2013;2:67. doi: 10.1186/2046-4053-2-67.

23. Zlotkin SH, Schauer C, Christofides A, Sharieff W, Tondeur MC, Hyder SMZ. Micronutrient sprinkles to control childhood anaemia. PLoS Med. 2005;2(1): e1. doi:10.1371/journal.pmed.0020001.

24. Neufeld, L. M., Sotres Alvarez, D., Gertler, P. J., Tolentino Mayo, L., Jiménez Ruiz, J., Fernald, L. C., et al. Impacto de Oportunidades en el crecimiento y estado nutricional de niños en zonas rurales. En B. H. Prado, and M. H. Ávila (Eds.), Evaluación externa de impacto del Programa Oportunidades 2004: Tomo III. Alimentación (pp. 17-52). Cuernavaca, México: Instituto Nacional de Salud Pública; 2005.

25. Leroy JL, Garcia-Guerra A, Garcia R, Dominguez C, Rivera J, Neufeld LM: The Oportunidades program increases the linear growth of children enrolled at young ages in urban Mexico. J Nutrition. 2008;138(4):793-798.

26. Gutiérrez, J. P., Bautista, S., Gertler, P., Hernández, M., \& Bertozzi, S. M: Impacto de Oportunidades en el estado de salud, morbilidad y utilización de servicios de salud de la población beneficiaria en zonas urbanas. En M. H. Ávila, B. H. Prado, \& J. E. U. Salomón (Eds.), Evaluación externa de impacto del Programa Oportunidades 2006 (pp. 15-52). Cuernavaca, México: Instituto Nacional de Salud Pública; 2006.

27. Sprinkles Global Health Initiative(SGHI) Micronutrient Sprinkles for use in Infants and Young Children: Guidelines on Recommendations for Use and Program Monitoring and Evaluation. Toronto, CA; 2008.

28. Khandker, S; Koolwal, G; Samad, H. Handbook on impact evaluation: quantitative methods and practices [Internet]. Washington DC: World Bank; 2011. [citado el 20 de octubre de 2018]. Disponible en: https://openknowledge.worldbank.org/bitstream/handle/10986/2693/520990PUB0EPI1 101 OfficialoUse0Only1. pdf ? sequence $=1$

Correspondencia: Juan Pablo Aparco Balboa. Dirección: Jr. Tizón y Bueno 276 Jesús María. Lima, Perú.

Teléfono: (+51) 748-0000 anexo 6626.

Email:japarco@ins.gob.pe

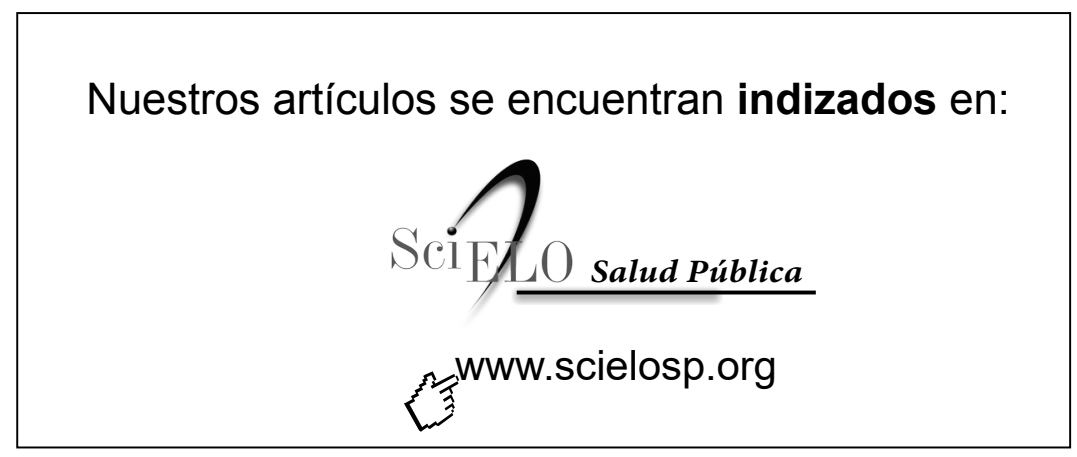

